OPEN ACCESS

Citation: Rizwana Zulfiqar, Kamran Habib, Memoona Khan, Abdul Nasir Khalid (2020) A taxonomic and phylogenetic study of some Lecidella species from Pakistan. Webbia. Journal of Plant Taxonomy and Geography 75(2): 219-230. doi: 10.36253/jopt-9581

Received: August 8, 2020

Accepted: September 9, 2020

Published: November 18, 2020

Copyright:@2020RizwanaZulfiqar, Kamran Habib, Memoona Khan, Abdul Nasir Khalid. This is an open access, peer-reviewed article published by Firenze University Press (http://www. fupress.com/webbia) and distributed under the terms of the Creative Commons Attribution License, which permits unrestricted use, distribution, and reproduction in any medium, provided the original author and source are credited.

Data Availability Statement: All relevant data are within the paper and its Supporting Information files.

Competing Interests: The Author(s) declare(s) no conflict of interest.

Editor: Riccardo M. Baldini

\section{A taxonomic and phylogenetic study of some Lecidella species from Pakistan}

\author{
Rizwana Zulfiqar ${ }^{\star}$, Kamran Habib, Memoona Khan, Abdul Nasir \\ KHALID \\ Fungal Biology and Systematics Lab, Department of Botany, University of the Punjab, \\ Quaid-e-Azam Campus, Lahore, 54590, Pakistan \\ ${ }^{\star}$ Corresponding author. Email: rizwanamughal6@gmail.com
}

\begin{abstract}
In this study, a number of species of the genus Lecidella were collected from different areas of Pakistan and characterized using morpho-anatomical and molecular techniques. The present work revealed that collected specimens belong to four species of Lecidella. Among which L. tumidula is a new record for Pakistan while L. carpathica, L. patavina and L. stigmatea are being reported here from new localities, from Pakistan, representing their wider distribution. Complete morpho-anatomical descriptions, ecology and distribution, along with ITS-based molecular analysis is provided.
\end{abstract}

Keywords: Khyber Pakhtunkhwa, Lecanoraceae, Lecidella, phylogeny, Pakistan.

\section{INTRODUCTION}

The genus Lecidella Korb. (Lecanoraceae), established by Korber in 1855, is comprised of 80 species (Zhao et al. 2015). This genus of crustose lichens is mainly characterized by black lecidiene apothecia with persistent proper excipulum and Lecidella-type asci (Zhao et al. 2015). It is a cosmopolitan genus, frequently found in temperate latitudes on wood, bark or rock (Kantvilas and Elix 2013). This group of lichenized ascomycetous fungi is usually regarded as taxonomically difficult due to a high degree of variation in morphological characters. In such cases, molecular study has played a significant role in systematics and species delimitation (Zhao et al. 2015).

Pakistan is located in western South Asia between $24-37^{\circ} \mathrm{N}$ latitude and $62-75^{\circ} \mathrm{E}$ longitudes. The country is well known for its geographical and climatic variations which is linked with rich biodiversity (IUCN 2006). The lichen diversity in this region is probably very high but little known due to the lack of surveys in many areas (Ahmad et al. 1997; Aptroot and Iqbal 2012). So far, 375 lichen species have been reported from Pakistan (Ahmad 1965; Aptroot and Iqbal 2012; Habib et al. 2017; Khan et al. 2018; Habib and Khalid 2019). In the past, attempts were made to describe the lichen diversity of the country using morpho-anatomical techniques (Ahmad 1965; Iqbal et al. 1978; Aptroot and Iqbal 2012). Recently, molecular techniques have also 
been used to study lichen flora of Pakistan (Habib et al. 2017; Khan et al. 2018; Habib and Khalid 2019).

From Pakistan, five species of Lecidella have been reported so far, viz; L. carpathica Korb., L. euphorea (Florke) Hertel, L. pulveraceae (Florke) Th.Fr., L. patavina (A.Massal.) Knoph \& Leuckert, and L. stigmatea (Ach.) Hertel \& Leuckert (Aptroot and Iqbal 2012). In the present study, different species of Lecidella have been collected from different areas of Pakistan. Use of morpho-anatomical techniques along with phylogenetic analysis led to identification of four different species. $L$. tumidula (A.Massal.) Knoph \& Leuckert has been collected and described for the first time from Pakistan which made an addition to the lichen flora of this country. Now, the number of Lecidella species reported from Pakistan has been raised from five to six.

\section{MATERIALS AND METHODS}

\section{Morphological and chemical studies}

Collections were made during a lichen survey of Chikar, Muzaffarabad (Azad Jammu and Kashmir), Parachinar, and Fairy Meadows (Gilgit Baltistan) in 2017 and 2018. Morphological characters were observed under a stereomicroscope (Meiji Techno, EMZ-5TR, Japan). Standard microscopy and spot tests (Hale 1979) were used for identification. Measurements were made from free hand section of apothecia mounted in water on a glass slide. The sections were observed using a compound microscope (MX4300H, Meiji Techno Co., Ltd., Japan). Minimum twenty measurements in water were made for each diagnostic feature.

\section{DNA extraction and PCR amplification}

We used thallus material along with apothecial material to extract fungal DNA using a $2 \%$ CTAB protocol (Gardes and Bruns 1993). The primer pair ITS1F (Grades and Bruns 1993) and ITS4 (White et al. 1990) was used to amplify the internal transcribed spacer (ITS) region under PCR conditions used by Khan et al. (2018). PCR products were visualized in a $1 \%$ agarose gel using ethidium bromide (Sambrook and Russell 2001). PCR products were sequenced from BGI, China.

\section{Phylogenetic analysis}

The ITS regions of all specimens were amplified and sequenced. Bio-edit sequence alignment editor was used to reassemble forward and reverse sequences (Hall 2005). Sequences of other Lecidella species based on initial BLAST searches and those used in a study on phylogeny of Lecidella by Zhao et al. (2015) were used in phylogenetic analysis (Table 1). The multiple sequence alignment was performed using MAFFT v7 with all parameters set to default values (Katoh and Standley 2013). The ends of the alignment were trimmed to nearly an equal number of sites for all sequences. All gaps were treated as missing data. Maximum Likelihood analysis was performed with MEGA6 using a GTR model for bootstrapping (Tamura et al. 2013). One thousand rapid bootstrap replicates were run to infer the evolutionary history of each species.

The length of the final aligned file was 541 nucleotides, of which 355 sites were conserved, 181 were variable, 156 were parsimony informative and 25 were singleton, Rhizoplaca porterii and R. parilis (HM577376, HM577309) were chosen as an outgroup (Zhao et al. 2015).

\section{RESULTS}

Lecidella tumidula (A.Massal.) Knoph \& Leuckert, Biblthca Lichenol. 68: 131. 1997. (Figure 1, A-E; Figure 5; A-D).

\section{Description}

Thallus crustose, continuous, up to $3 \mathrm{~cm}$ in diameter, up to $0.6 \mathrm{~mm}$ thick, granulose to rimose. Colour: light greyish green to light olive green, dull to weakly glossy. Apothecia: rounded, semi-immersed, sessile, 0.5$1 \mathrm{~mm}$, frequently present, strongly constricted at base. Margins: thin, continuous, concolorous to disc, distinct when young, indistinct when mature. Disc: black, smooth, glossy, pruinose, flat to convex. Exciple: black, 40-50 $\mu \mathrm{m}$ thick. Epihymenium: blackish, 30-36 $\mu \mathrm{m}$ tall. Hymenium: hyaline, $80-86 \mu \mathrm{m}$ tall. Hypothecium: dark reddish brown, $30-40 \mu \mathrm{m}$ tall. Paraphyses: aseptate, hyaline, rarely anastomosing or branched, apex swollen, 2-4 $\mu \mathrm{m}$ wide. Asci: clavate, 8 -spored, $58-78 \mu \mathrm{m} \times 10-14 \mu \mathrm{m}$. Ascospores: simple, hyaline, narrowly ellipsoid to ovoid, $10-12 \mu \mathrm{m} \times 5-7 \mu \mathrm{m}$.

\section{Spot Tests}

All negative (diploicin and lichexanthone reported according to literature but not examined in our specimen).

\section{Substrate and ecology}

L. tumidula was found on bark of Quercus incana W. Bartram in dry temperate forest, at an altitude of 1,705 
Table 1. Specimens used in phylogenetic analysis of Lecidella species.

\begin{tabular}{|c|c|c|c|c|c|c|c|}
\hline $\begin{array}{l}\text { ITS Accession } \\
\text { No- }\end{array}$ & $\begin{array}{l}\text { Specimen } \\
\text { Name }\end{array}$ & Country & Voucher No- & $\begin{array}{l}\text { ITS Accession } \\
\text { No- }\end{array}$ & $\begin{array}{l}\text { Specimen } \\
\text { Name }\end{array}$ & Country & Voucher No- \\
\hline KT453736 & $\begin{array}{l}\text { Lecidella } \\
\text { tumidula }\end{array}$ & China & $\begin{array}{c}\text { ZX XL0009 } \\
\text { (Zhao et al. 2015) }\end{array}$ & MK970672 & $\begin{array}{l}\text { Lecidella } \\
\text { greenii }\end{array}$ & Antarctica & $\begin{array}{c}\text { T48787 } \\
\text { (Wagner et al. 2019) }\end{array}$ \\
\hline KT453737 & $\begin{array}{l}\text { Lecidella } \\
\text { tumidula }\end{array}$ & China & $\begin{array}{l}\text { ZX 20129166-2 } \\
\text { (Zhao et al. 2015) }\end{array}$ & HQ605934 & $\begin{array}{l}\text { Lecidella } \\
\text { patavina }\end{array}$ & Turkey & $\begin{array}{c}574971 \\
\text { (Basaran et al. 2014) }\end{array}$ \\
\hline HQ650596 & $\begin{array}{l}\text { Lecidella } \\
\text { tumidula }\end{array}$ & USA & $\begin{array}{c}404720 \\
\text { (Schmull et al. 2011) }\end{array}$ & KT453767 & $\begin{array}{l}\text { Lecidella } \\
\text { patavina }\end{array}$ & China & $\begin{array}{l}\text { ZX 20140501-2 } \\
\text { (Zhao et al. 2015) }\end{array}$ \\
\hline KT453750 & $\begin{array}{c}\text { Lecidella } \\
\text { elaeochromoid }\end{array}$ & China & $\begin{array}{c}\text { ZX 20141142 } \\
\text { (Zhao et al. 2015) }\end{array}$ & MK620163 & $\begin{array}{l}\text { Lecidella } \\
\text { stigmatea }\end{array}$ & Argentina & $\begin{array}{c}\text { UR00128 } \\
\text { (Ruprecht et al. 2019) }\end{array}$ \\
\hline KT453746 & $\begin{array}{c}\text { Lecidella } \\
\text { elaeochromoid }\end{array}$ & China & $\begin{array}{l}\text { ZX 20114966-2 } \\
\text { (Zhao et al. 2015) }\end{array}$ & MK620136 & $\begin{array}{l}\text { Lecidella } \\
\text { stigmatea }\end{array}$ & Argentina & $\begin{array}{c}\text { UR00080 } \\
\text { (Ruprecht et al. 2019) }\end{array}$ \\
\hline KT453748 & $\begin{array}{l}\text { Lecidella } \\
\text { effugiens }\end{array}$ & China & $\begin{array}{l}\text { ZX 20141269-2 } \\
\text { (Zhao et al. 2015) }\end{array}$ & KT453764 & $\begin{array}{l}\text { Lecidella } \\
\text { stigmatea }\end{array}$ & China & $\begin{array}{l}\text { ZX 20140086-2 } \\
\text { (Zhao et al. 2015) }\end{array}$ \\
\hline KT453747 & $\begin{array}{l}\text { Lecidella } \\
\text { effugiens }\end{array}$ & China & $\begin{array}{l}\text { ZX 20141148-2 } \\
\text { (Zhao et al. 2015) }\end{array}$ & KT453765 & $\begin{array}{l}\text { Lecidella } \\
\text { stigmatea }\end{array}$ & China & $\begin{array}{l}\text { ZX 20140045-2 } \\
\text { (Zhao et al. 2015) }\end{array}$ \\
\hline AF517929 & $\begin{array}{l}\text { Lecidella } \\
\text { meiococca }\end{array}$ & Sweden & $\begin{array}{c}\text { Ekman } 3101(\mathrm{BG}) \\
\text { (Ekman \& Tonsberg, 2002) }\end{array}$ & KT453760 & $\begin{array}{l}\text { Lecidella } \\
\text { stigmatea }\end{array}$ & China & $\begin{array}{l}\text { ZX 20140519-2 } \\
\text { (Zhao et al. 2015) }\end{array}$ \\
\hline KT453743 & $\begin{array}{l}\text { Lecidella } \\
\text { euphorea }\end{array}$ & China & $\begin{array}{c}\text { ZX XL0387 } \\
\text { (Zhao et al. 2015) }\end{array}$ & KT453763 & $\begin{array}{l}\text { Lecidella } \\
\text { stigmatea }\end{array}$ & China & $\begin{array}{l}\text { ZX 20140507-2 } \\
\text { (Zhao et al. 2015) }\end{array}$ \\
\hline KT453742 & $\begin{array}{l}\text { Lecidella } \\
\text { euphorea }\end{array}$ & China & $\begin{array}{c}\text { ZX 20140638 } \\
\text { (Zhao et al. 2015) }\end{array}$ & KT695322 & $\begin{array}{l}\text { Lecidella } \\
\text { carpathica }\end{array}$ & Canada & $\begin{array}{l}\text { BIOUG24047-H06 } \\
\text { (Telfer et al. 2015) }\end{array}$ \\
\hline KX132994 & $\begin{array}{c}\text { Lecidella } \\
\text { flavosorediata }\end{array}$ & Switzerland & $\begin{array}{c}980812 \\
\text { (Mark et al. 2016) }\end{array}$ & KT695353 & $\begin{array}{l}\text { Lecidella } \\
\text { carpathica }\end{array}$ & Canada & $\begin{array}{l}\text { BIOUG24047-E02 } \\
\text { (Telfer et al. 2015) }\end{array}$ \\
\hline KX132965 & $\begin{array}{c}\text { Lecidella } \\
\text { flavosorediata }\end{array}$ & Switzerland & $\begin{array}{c}980812 \\
\text { (Mark et al. 2016) }\end{array}$ & JN873899 & Lecidella siplei & Antarctica & $\begin{array}{c}\text { Tuerk } 35895 \\
\text { (Ruprecht } \text { et al. 2012) }\end{array}$ \\
\hline JN873903 & $\begin{array}{c}\text { Lecidella } \\
\text { wulfenii }\end{array}$ & Austria & $\begin{array}{c}\text { Tuerk } 39666 \\
\text { (Ruprecht et al. 2012) }\end{array}$ & JN873896 & Lecidella siplei & Antarctica & $\begin{array}{c}\text { Tuerk } 32991 \\
\text { (Ruprecht } \text { et al. 2012) }\end{array}$ \\
\hline MN387031 & $\begin{array}{c}\text { Lecidella } \\
\text { elaeochroma }\end{array}$ & Poland & $\begin{array}{c}272987 \\
\text { (Singh et al. 2019) }\end{array}$ & JN873898 & Lecidella siplei & Antarctica & $\begin{array}{c}\text { Tuerk } 33457 \\
\text { (Ruprecht et al. 2012) }\end{array}$ \\
\hline MN387029 & $\begin{array}{c}\text { Lecidella } \\
\text { elaeochroma }\end{array}$ & Poland & $\begin{array}{c}272987 \\
\text { (Singh et al. 2019) }\end{array}$ & JN873897 & Lecidella siplei & Antarctica & $\begin{array}{c}\text { Tuerk } 33449 \\
\text { (Ruprecht et al. 2012) }\end{array}$ \\
\hline HQ287871 & $\begin{array}{l}\text { Lecidella } \\
\text { greenii }\end{array}$ & Antarctica & $\begin{array}{l}\text { Herbarium Tuerk } 43015 \\
\text { (Lumbsch et al. 2011) }\end{array}$ & HM577376 & $\begin{array}{l}\text { Rhizoplaca } \\
\text { porterii }\end{array}$ & USA & $\begin{array}{c}55145 \text { (BRY-C) } \\
\text { (Leavitt et al. 2011) }\end{array}$ \\
\hline JN873884 & $\begin{array}{l}\text { Lecidella } \\
\text { greenii }\end{array}$ & Antarctica & $\begin{array}{c}\text { Tuerk } 33612 \\
\text { (Ruprecht } \text { et al. 2012) }\end{array}$ & HM577309 & $\begin{array}{l}\text { Rhizoplaca } \\
\text { parilis }\end{array}$ & USA & $\begin{array}{c}55078 \text { (BRY-C) } \\
\text { (Leavitt et al. 2011) }\end{array}$ \\
\hline
\end{tabular}

$\mathrm{m}$, dominant vegetation including Pinus gerardiana Wall. ex D. Don, Quercus ilex L., Juniperus macropoda Boiss. and Picea smithiana Boiss, temperature ranges between $6-16{ }^{\circ} \mathrm{C}$, average annual rainfall $300-500 \mathrm{~mm}$.

\section{Distribution}

Temperate areas of Asia including China (Zhao et al. 2015), have also been reported from Europe and North America (Nash et al. 2004). Here it is been reported for the first time from Pakistan.

\section{Material examined}

PAKISTAN: Khyber Pakhtunkhwa Province: Kurram District, Parachinar: 3390' N, 7008' E; 1,705 m; on bark of Quercus incana W. Bartram, 18 August 2018,
A.N. Khalid \& K. Habib; LAH36399; (GenBank accession no. MT139651).

Lecidella carpathica Korb., Parerga lichenol. (Breslau) 3: 212. 1861. (Figure 2, A-F; Figure 5; E-H).

\section{Description}

Thallus crustose, effuse, rimose areolate to subsquamulose, dull, up to $5 \mathrm{~cm}$ wide, up to $300 \mu \mathrm{m}$ thick. Areoles: scattered to crowded, smooth or rough, up to $0.5 \mathrm{~mm}$ in diameter, irregular in outline. Colour: pale green to grey green. Apothecia: sessile, semi-immersed, constricted at base, scattered to crowded, up to $1 \mathrm{~mm}$ in diameter. Disc: black, plane, flat to moderately con- 

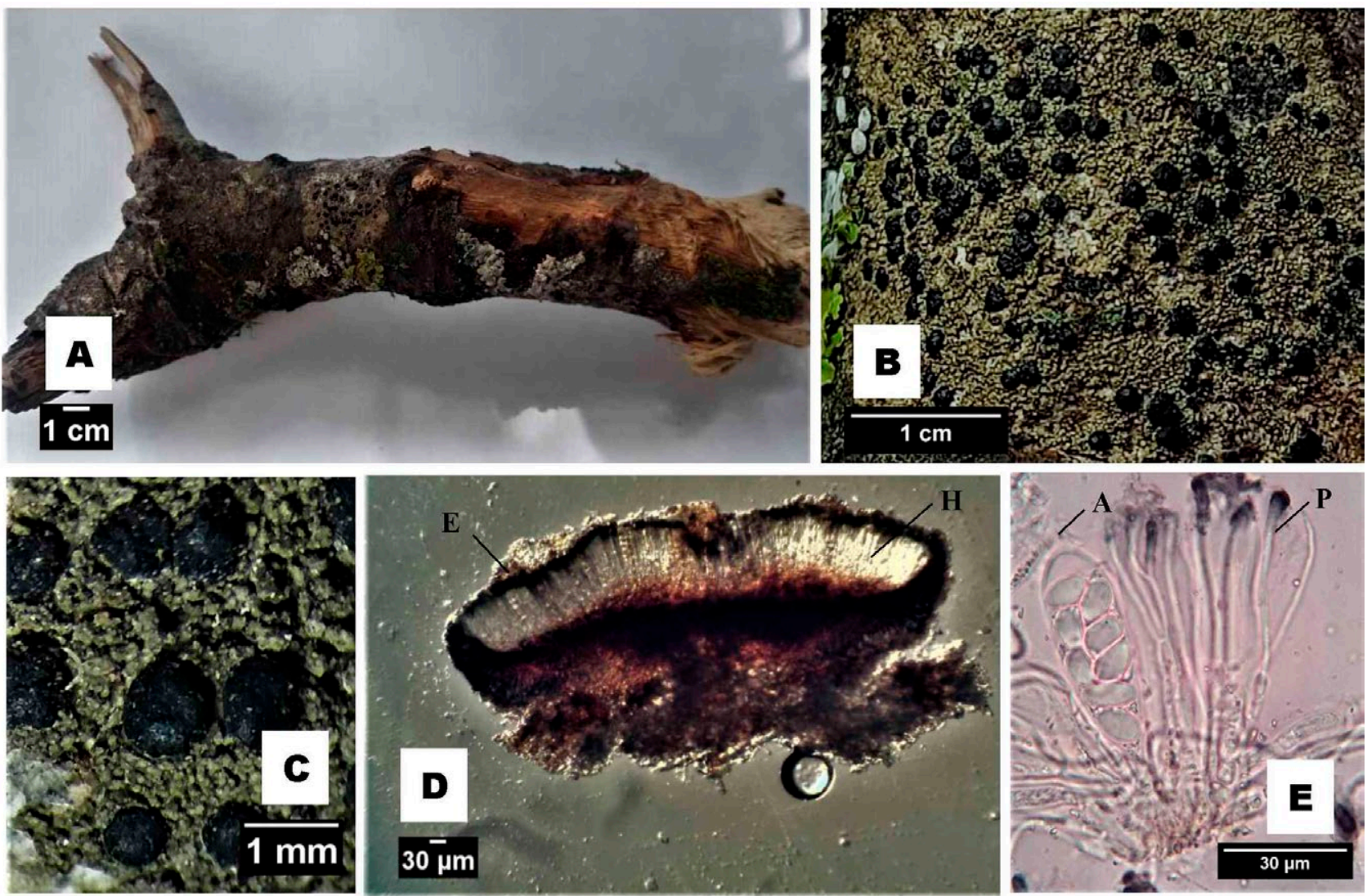

Figure 1. Lecidella tumidula. (A \& B) showing crustose thallus and abundant apothecia (C) pruinose apothecia; (D) Cross section of an apothecium (H: Hymenium; E: Epihymenium); (E) showing ascus and paraphyses (A: Ascus; P: Paraphyses).

vex, pruinose. Margins: distinct, entire or flexuose, up to $0.9 \mathrm{~mm}$ wide. Exciple: bluish green to black, 20-30 $\mu \mathrm{m}$ thick. Epihymenium: pale brown to dark brown, 15-20 $\mu \mathrm{m}$ tall. Hymenium: hyaline, 65-90 $\mu \mathrm{m}$ tall. Hypothecium: pale brown to brown, 30-45 $\mu \mathrm{m}$ tall. Paraphyses: aseptate, hyaline, rarely anastomosing or branched, sometimes slightly swollen apically, up to $2 \mu \mathrm{m}$ wide. Asci: clavate, $45-60 \times 13-15 \mu \mathrm{m}, 8$-spored. Ascospores: simple, hyaline, ellipsoid to ovoid, $10-14 \times 6-8 \mu \mathrm{m}$.

Spot tests

$$
\mathrm{K}+\text { yellow, C-, KC-, P- }
$$

Substrate and ecology

L. carpathica was found on rock (saxicolous), in moist temperate forest at an altitude of $234 \mathrm{~m}$, temperature ranges between $-2-37{ }^{\circ} \mathrm{C}$, average annual rainfall 1,500-1,600 mm, with dominant tree species Pinus wallichiana A.B. Jacks., Picea smithiana Boiss., Abies pindrow Royle., Quercus incana W. Bartram and Q. dilatata Lindl. ex Royle.

\section{Distribution}

Widespread including Africa, Europe, Australia, Macaronesia, New Zealand (Smith et al. 2009), UK, USA, Ukraine (Oxner 1968; Kondratyuk et al. 1998; 2003), temperate parts of Asia (Nash et al. 2004), China (Zhao et al. 2015), India (Singh \& Sinha 2010) and Pakistan (Nasim et al. 2004).

\section{Material examined}

PAKISTAN: Azad Jammu \& Kashmir: Chikar, $34^{\circ} 9^{\prime} \mathrm{N}, 73^{\circ} 41^{\prime} \mathrm{E}, 234 \mathrm{~m}$; on rock; 18 August 2018, $\mathrm{K}$. Habib; LAH36400; (Genbank accession no. MT139649).

Lecidella patavina (A.Massal.) Knoph \& Leuckert, in Knoph, Bibliotheca Lichenol. 36: 116. 1990. (Figure 3, A-G; Figure 6; A-D).

\section{Description}

Thallus indistinctly crustose, inconspicuous, intermingled with rock particles, up to $4 \mathrm{~cm}$ in diameter. 

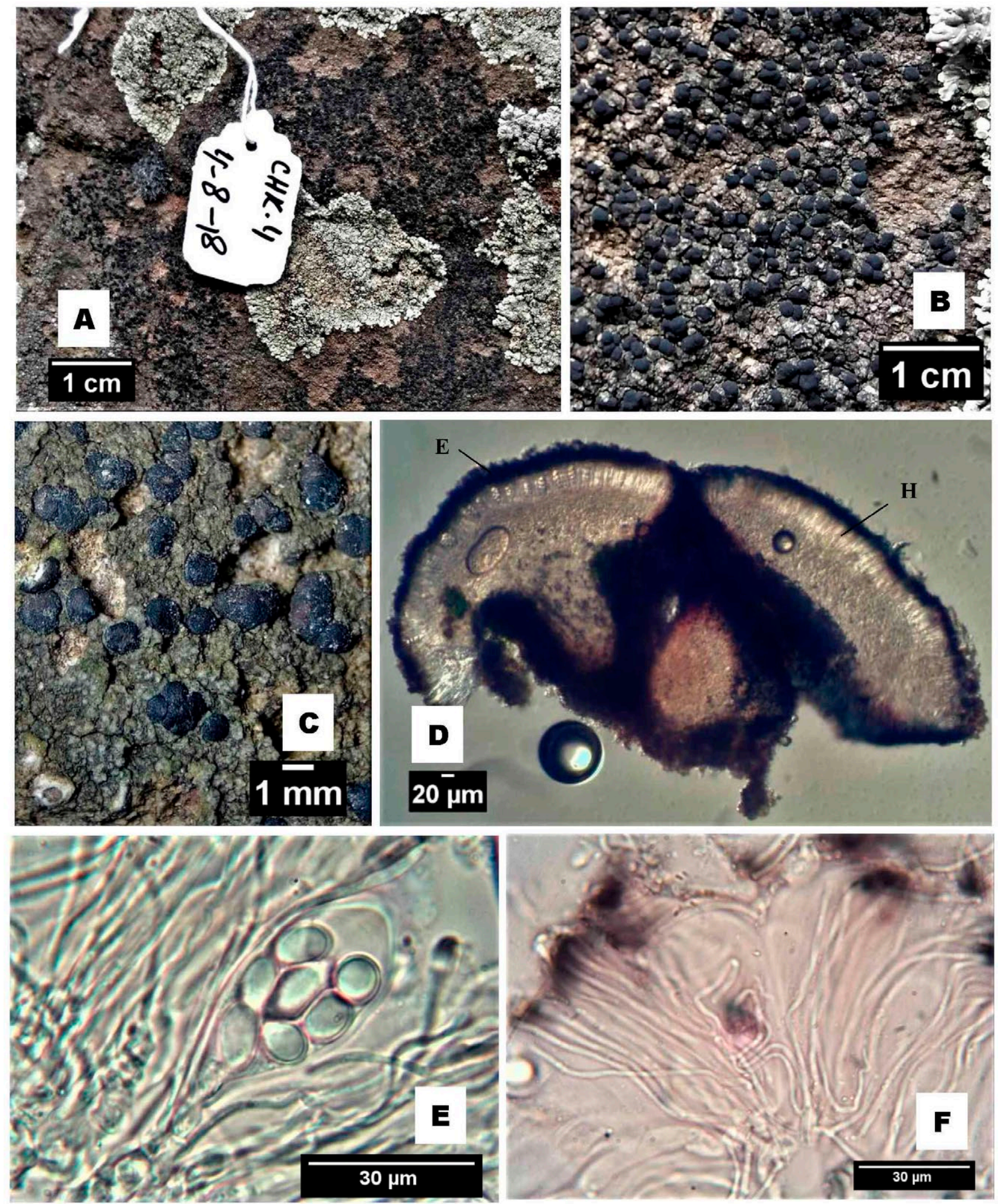

Figure 2. Lecidella carpathica. (A \& B) Crustose thallus showing abundant apothecia; (C) black, pruinose apothecia; (D) Cross section of an apothecium (H: Hymenium; E: Epihymenium); (E) Ascus; (F) Paraphyses. 

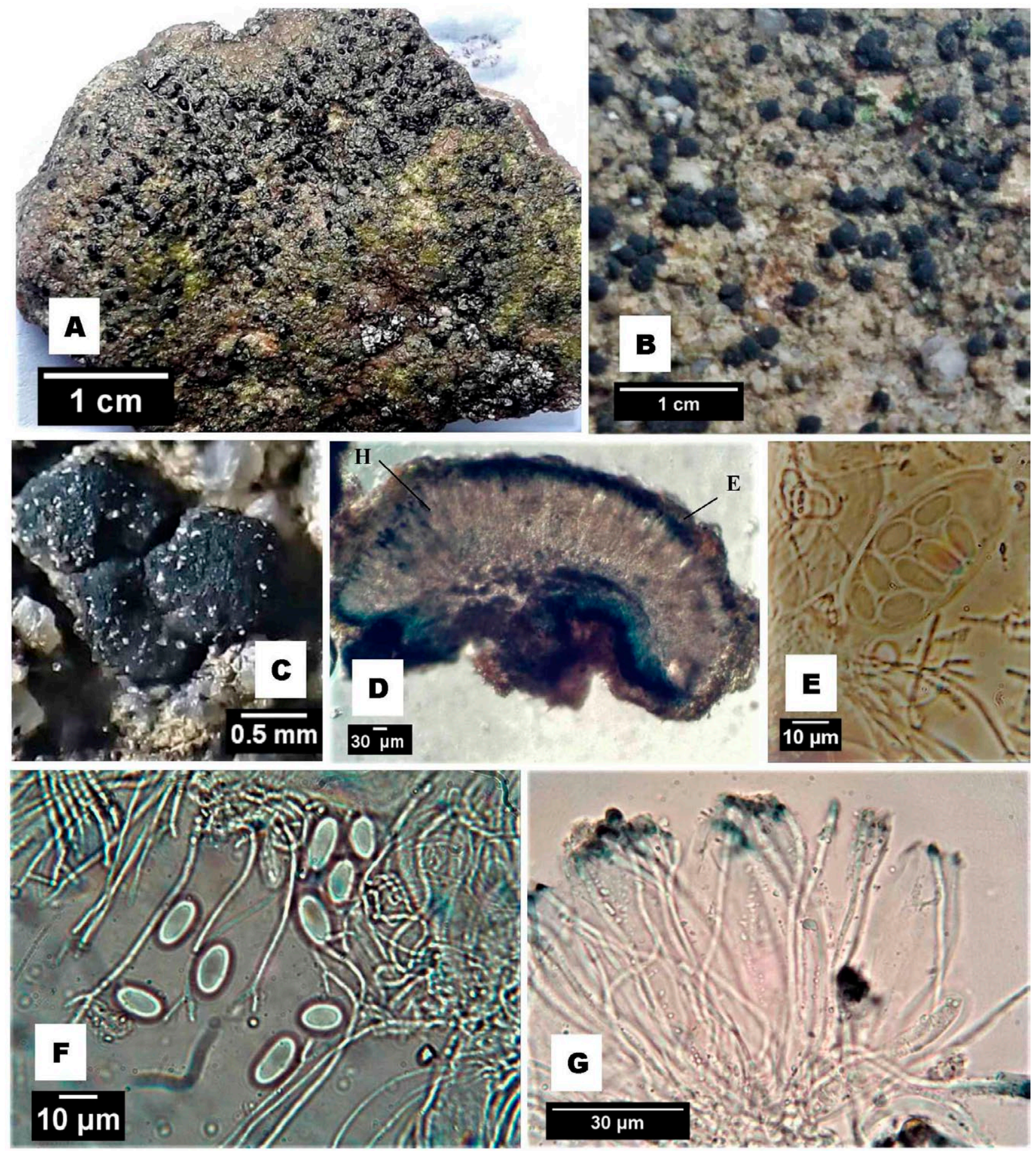

Figure 3. Lecidella patavina. (A \& B) Crustose thallus showing abundant apothecia; (C) black, pruinose fascicle of apothecia; (D) Cross section of an apothecium (H: Hymenium; E: Epihymenium); (E) Ascus; (F) Ascospores; (G) Paraphyses.

Colour: light green to dark green. Apothecia: rounded to irregular, $0.2-1.5 \mathrm{~mm}$ in diameter, sessile, constricted at base, frequent, separate, rarely fascicle. Margins: thin, continuous, concolorous to disc, distinct when young, indistinct when mature. Disc: black, flat to strongly convex, smooth and slightly glossy, slightly pruinose. Exci- 
ple: bluish green, $40-50 \mu \mathrm{m}$ thick. Epihymenium: dark blue with blackish tint, $20-30 \mu \mathrm{m}$ tall. Hymenium: hyaline to grey, $110-120 \mu \mathrm{m}$ tall, inspersed. Hypothecium: hyaline to brown, $90-110 \mu \mathrm{m}$ tall. Paraphyses: hyaline, aseptate, apically branched, not anastomosing, apex slightly swollen, 1-2 $\mu \mathrm{m}$ wide. Asci: clavate, 8-spored, 30-67 $\mu \mathrm{m} \times 12-20 \mu \mathrm{m}$. Ascospores: hyaline, simple, narrowly ellipsoid to ovoid, $9-13 \mu \mathrm{m} \times 5-6 \mu \mathrm{m}$.

Spot Test

$\mathrm{K}+$ (Slight yellow), C-, KC-, P- (atranonin or lichexanthone might be predicted).

\section{Substrate and ecology}

L. patavina was found on nutrient enriched siliceous rocks near water falls, in moist temperate forest at an altitude of $1,705 \mathrm{~m}$, temperature ranges between $-2-37$ ${ }^{\circ} \mathrm{C}$, average annual rainfall $1,500-1,650 \mathrm{~mm}$, with dominant tree species Cedrus deodara (Roxb. ex Lambert) G.Don, Pinus wallichiana A.B. Jacks., Picea smithiana Boiss., Abies pindrow Royle., Quercus incana W.Bartram, Q. dilatata Lindl. ex Royle. and Q. semecarpifolia Sm.

\section{Distribution}

Mainly arctic-alpine, UK, N.W. Scotland (Smith et al. 2009), Africa, Antarctica, Europe, USA, (Knoph and Leuckert 2004; Knudsen and Kocourková 2012), Ukraine (Vondrak et al. 2010), temperate parts of Asia (Nash et al. 2004), China (Zhao et al. 2015) and Pakistan (Aptroot and Iqbal 2012).

\section{Material examined}

PAKISTAN: Khyber Pakhtunkhwa Province: Kurram District, Parachinar: $33^{\circ} 90^{\prime} \mathrm{N}, 70^{\circ} 08^{\prime} \mathrm{E}$; $1705 \mathrm{~m}$; on rock; 18 August 2018, A.N. Khalid \& K. Habib; LAH3640; (GenBank accession no. MT139652).

Lecidella stigmatea (Ach.) Hertel \& Leuckert, Willdenowia 5: 375. 1969. (Figure 4, A-G; Figure 6; E-H).

\section{Description}

Thallus crustose, areolate or rimose-areolate to verruculose-areolate, $7-8 \mathrm{~cm}$ in diameter, up to $0.2 \mathrm{~mm}$ thick, rarely thick up to $0.6 \mathrm{~mm}$. Areoles: indistinct to distinct, flat to slightly convex, angular to irregular in outline, $0.2-0.6 \mathrm{~mm}$ in diameter. Surface: finely granulose, rough, dull. Colour: greyish to grey to brownish grey. Apothecia: lecideine, black, sessile, $0.4-0.9 \mathrm{~mm}$ in diameter. Disc: black, flat to somewhat convex, pruinose. Margins: distinct, thin, becoming excluded. Exciple:
78-88 $\mu \mathrm{m}$ thick, bluish green to brown. Epihymenium: dark brown, $12-16 \mu \mathrm{m}$ high. Hymenium: hyaline, 75-80 $\mu \mathrm{m}$ tall, not inspersed. Hypothecium: hyaline to brown, 65-75 $\mu \mathrm{m}$ tall. Paraphyses: aseptate, hyaline, rarely anastomosing or branched, slightly swollen apically, up to 1.2 $\mu \mathrm{m}$ wide. Asci: lecanoral-type, clavate, 8-spored, 40-57 $\mathrm{x}$ 14-18 $\mu \mathrm{m}$; Ascospores: simple, hyaline, thick and smooth wall, ellipsoid to ovoid, 9-14 x 5-8 $\mu \mathrm{m}$.

Spot tests

$\mathrm{K}+$ yellow, C-, KC-, P- (atranonin, zeorin, lichexanthone or norlichexanthone might be predicted).

\section{Substrate and ecology}

L. stigmatea was found on bark of Pinus wallichiana A.B. Jacks., in moist temperate forest, with dominant tree species Cedrus deodara (Roxb. ex Lambert) G.Don, Pinus wallichiana A.B. Jacks., Picea smithiana Boiss., Abies pindrow Royle., Quercus incana W. Bartram, Q. dilatata Lindl. ex Royle. and Q. semecarpifolia Sm. at an altitude of 3,300 $\mathrm{m}$, temperature ranges between -2-37 ${ }^{\circ} \mathrm{C}$, average annual rainfall $1650 \mathrm{~mm}$.

The second collection of L. stigmatea was found on a rock (saxicolous), close to water falls, in moist temperate forest at an altitude of $2,900 \mathrm{~m}$, temperature ranges between $-2-37^{\circ} \mathrm{C}$, average annual rainfall 1,500-1,650 $\mathrm{mm}$.

\section{Distribution}

Probably cosmopolitan, including UK, USA, Antarctica, Australia (Smith et al. 2009), Ukraine (Oxner 1968, Kondratyuk et al. 1998, 2003), temperate parts of Asia (Nash et al. 2004), China (Zhao et al. 2015), India (Singh \& Sinha 2010) and Pakistan (Ahmad 1965).

\section{Material examined}

PAKISTAN: Gilgit Baltistan, Fairy Meadows: $35^{\circ} 23^{\prime}$ N, $74^{\circ} 35^{\prime}$ E; $3300 \mathrm{~m}$; on bark of Pinus wallichiana A.B. Jacks., 18 July 2017, A.N. Khalid \& M. Khan; LAH36402; (GenBank accession no. MT139653); Azad Jammu \& Kashmir: District Muzaffarbad, Peer Chanasi: $34^{\circ} 2^{\prime} \mathrm{N}$, 73³3' E; 2900 m; on rock; 18 August 2018, K. Habib; LAH36403; (Genbank accession no. MT139650).

Key to Genus Lecidella in Pakistan

1a. On Bark L. tumidula

1b. On rock, Cortex K-, C...………………………………..... 2

1c. On rock, or on bark, Cortex $\mathrm{K}+, \mathrm{C}$...................................... 3 

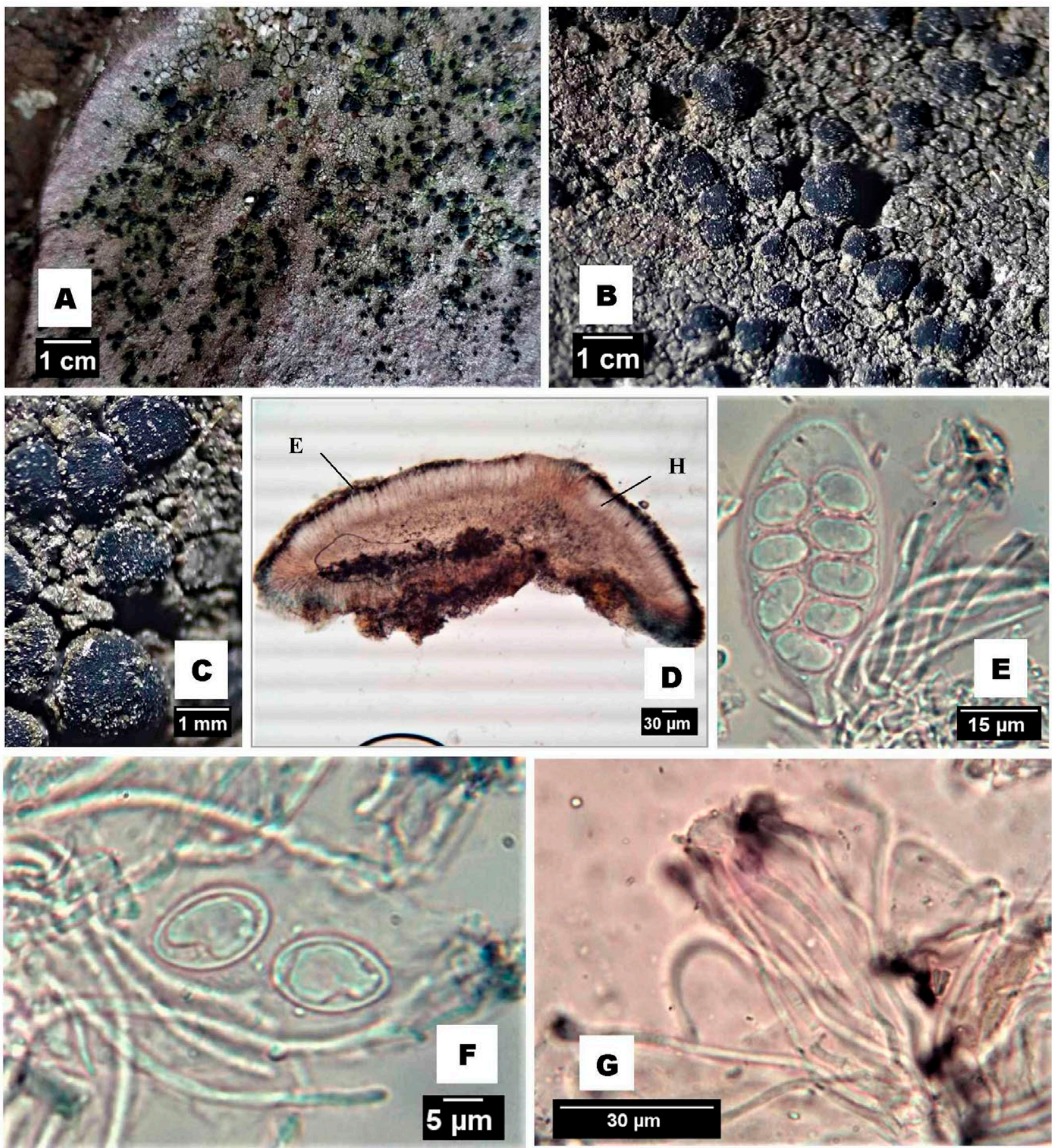

Figure 4. Lecidella stigmatea. (A \& B) Crustose thallus showing abundant apothecia; (C) black, pruinose disc of apothecia; (D) Cross section of an apothecium (H: Hymenium; E: Epihymenium); (E) Ascus; (F) Ascospores; (G) Paraphyses.

1d. On rock, Cortex $\mathrm{K}+$, C

2a. Thallus crustose, areolate, epihymenium 15-20 $\mu \mathrm{m}$ tall, hypothecium brownish grey, on rock 2b. Thallus crustose, granulose, epihymenium 30-36 $\mu \mathrm{m}$ tall, hypothecium dark reddish brown, on bark ....... L. tumidula

3a. Thallus rimose-areolate, paraphyses rarely branched, exciple greenish black, hymenium not inspersed ... L. stigmatea 

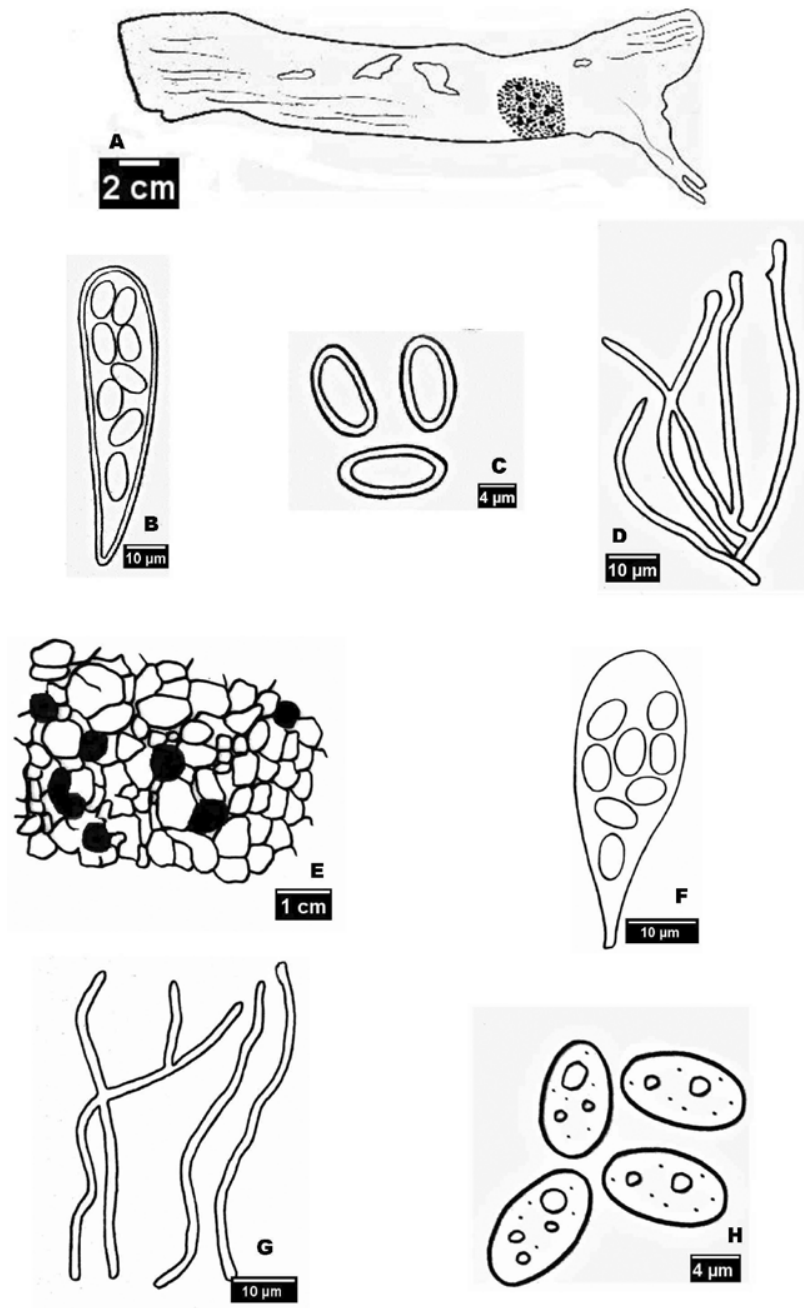

Figure 5. (A-D): Line drawings of Lecidella tumidula A: Granulose thallus; B: Ascus; C: Ascospores; D: Paraphyses; (E-H): Line drawings of Lecidella carpathica E: Crustose-areolate thallus; F: Ascus; G: Paraphyses; H: Ascospores.

3b. Thallus inconspicuous, paraphyses mostly branched, exciple bluish green, hymenium inspersed L. patavina

4a. Thallus crustose, granulose to rimose-areolate, hymenium 55-100 $\mu \mathrm{m}$ tall L. euphorea

4b. Thallus sorediate, farinose, hymenium $60-70 \mu \mathrm{m}$ tall L. pulveraceae

\section{Discussion}

The present study of the genus Lecidella from northern Pakistan revealed one new record for the lichen flora of Pakistan while other Lecidella species have been collected from new localities thus indicating their wide distributional range in Pakistan.
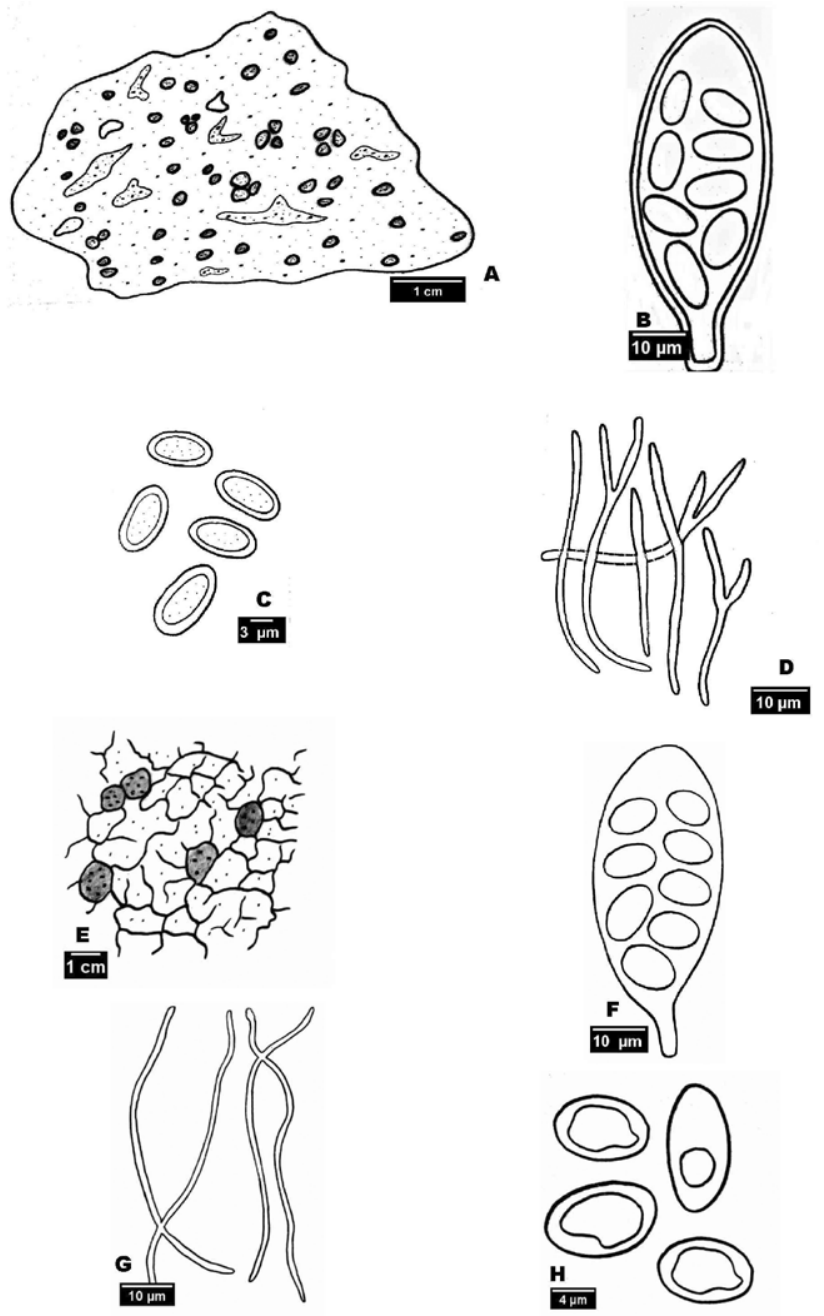

Figure 6. (A-D): Line drawings of Lecidella patavina A: crustose thallus B: Ascus; C: Ascospores; D: Paraphyses; (E-H): Line drawings of Lecidella stigmatea E: Rimose-areolate thallus; F: Ascus; G: Paraphyses; H: Ascospores.

In the ITS-based phylogenetic analysis, the Pakistani collection of L. tumidula (PR-112) clustered with specimens of the same taxon reported from China (Accession no. KT453736, KT453737) with strong support (99\%, Fig. 7) and formed a sister group relationship with L. tumidula collected from USA (Accession no. HQ650596) that was wrongly reported in GenBank (Schmull et al. 2011), but after re-examination it was L. tumidula (Zhao et al. 2015).

Morphological comparison also confirms its identity as L. tumidula (Nash et al. 2004) except the presence of a pruinose disc in the specimen collected from Pakistan. There is only one nucleotide difference between the Pakistani collection and the Chinese L. tumidula specimen. It is also the second report of this taxon from Asia after China (Zhao et al. 2015). 


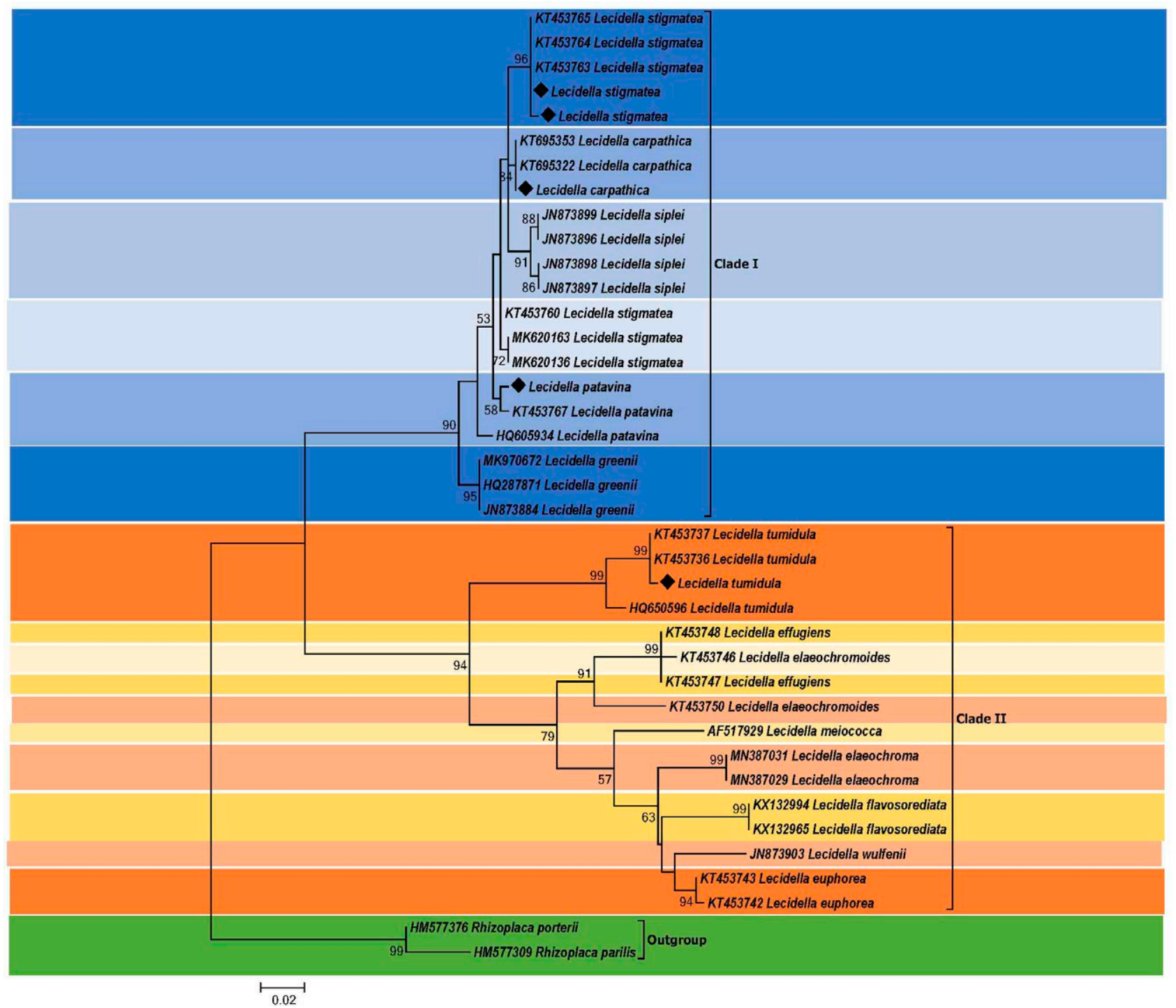

Figure 7. Phylogenetic analysis of species of Lecidella, comprised of 39 sequences. This tree has been inferred using maximum likelihood method. The bootstrap values based on 1000 replicates are shown below the branches. Sequences generated from local collection are marked with

In the phylogenetic analysis, the ITS sequence of Lecidella carpathica (CHK-04) clustered with ITS sequences of $L$. carpathica collected from Canada (KT695322, KT695353). Morphological comparison also confirms its identity as L. carpathica (Nash et al. 2004). There is only one nucleotide difference between Pakistani and Canadian L. carpathica (KT695322, KT695353). Previously, it was reported from dry temperate forest of Kalam (Swat), Pakistan at an elavation of about 2,001 m. a.s.l. The new collection is from moist temperate forest of AJK state, found at an elevation of 2,900 m. a.s.l.

The ITS sequence of Lecidella patavina (PR-11) clustered with a Chinese collection of L. patavina (Accession no. KT453767) in the phylogenetic analysis and made a sister branch with L. stigmatea (Ach.) Hertel \& Leuckert. Morphologically, both Lecidella species are similar in having the same chemistry, a crustose epilithic thallus with a black disc of apothecia (Basaran et al. 2014) but differ due to the presence of the conspicuous and thicker thallus in the latter.

There are also two nucleotide difference between Pakistani and Chinese collection of L. patavina (KT453767). From Pakistan, it was previously reported but locality was not recorded. Here it is described from high elevation in Parachinar i.e. 1,705 m. a.s.l., where it is widespread in warm and temperate climates. 
The ITS sequence of Lecidella stigmatea (MKF-7 and PC-34), clustered with L. stigmatea reported from China (KT453763, KT453764, KT453765). The L. stigmatea (MK620163, MK620136) in our tree formed separate subclade. Results from phylogenetic analysis of $L$. stigmatea requires revision between specimens identified as L. stigmatea from Argentina, are distantly related to samples of our clade. Morphologically our specimen is similar to the Sonoran L. stigmatea, in having rimose-areolate thallus and lecideine apothecia (Nash et al. 2004). From Pakistan, this taxon has previously been reported from dry temperate forest of Kalam (Swat), Pakistan, at an elavation of about 2,001 m. a.s.l. but recent collections are from moist temperate forests of Azad Jammu \& Kashmir and Gilgit Baltistan, at high elevations $2,900 \mathrm{~m}$ and 3,300 m. a.s.l. respectively.

\section{ACKNOWLEDGEMENTS}

We are sincerely thankful to Prof. Dr. Sci. Sergey Yakovych Kondratyuk, Kholodny Institute of Botany, National Academy of Sciences of Ukraine, for presubmission review of the manuscript and Dr. Francis Q. Brearley, School of Science and the Environment, Manchester Metropolitan University, for linguistic revision of the manuscript.

\section{REFERENCES}

Ahmad S. 1965. A preliminary contribution to the lichenflora of West Pakistan. Biologia (Lahore). 11: 21-47.

Aptroot A, Iqbal SH. 2012. Annotated checklist of the Lichens of Pakistan, with reports of new records. Herzogia. 25 (2): 211-229.

Başaran E, Cansaran-Duman D, Büyük İ, Aras S. 2014. Identification of some Lecidea, Porpidia and Lecidella species (lichen-forming ascomycetes) distributed in Turkey by sequence analysis of rDNA ITS region. Turkish hygiene and Experimental Biology Journal. 72 (1): 45-58.

Gardes M, Bruns TD. 1993. ITS primers with enhanced specificity for basidiomycetes-application to the identification of mycorrhizae and rusts. Molecular Ecology. 2 (2): 113-118.

Habib K, Khalid AN. 2019. New records of lichens from the State of Azad Jammu and Kashmir, Pakistan corroborated by ITS sequences. Nova Hedwigia. 109 (3-4): 457-473.

Habib K, Imran A, Khalid AN, Fiaz M. 2017. Some new records of lichens from Hunza valley, Pakistan. Pakistan Journal of Botany. 49 (6): 2475-2482.
Hale ME. 1979. How to know the Lichens (2nd ed.). Dubuque, Iowa: Wm. C. Brown Company Publishers. Pp. 246.

Hall TA. 2005. Bioedit Version 7.0.4. Department of Microbiology. North Carolina State University.

Iqbal SH, Bhatty SF, Gilani A. 1978. Macrolichens of Pakistan. 2 - genus Parmelia. Biologia (Lahore). 24: 140-173

IUCN [International Union of Conservation for Nature] 2006. News release: Pakistan's rich biodiversity faces serious threats. http://www.iucn.org.neral.

Kantvilas G, Elix JA. 2013. The lichen genus Lecidella (Lecanoraceae), with special reference to the Tasmanian species. Muelleria. 31: 31-47.

Katoh K, Standley DM. 2013. MAFFT multiple sequence alignment software version 7 , improvement in performance and usability. Molecular Biology and Evolution. 30: 772-780.

Khan M, Khalid AN, Lumbsch HT. 2018. A new species of Lecidea (Lecanorales, Ascomycota) from Pakistan. Mycokeys. 38: 25.

Knoph JG, Leuckert C. 2004. Lecidella. In: Nash T.H. III, Ryan B.D. Diederich P. Gries C. and. Bungartz F (eds.). - Lichen Flora of the Greater Sonoran Desert Region 2: 309-320. - Lichens Unlimited, Tempe, AZ. Desert Region. 2: 287-309.

Knudsen K, Kocourková J. 2012. The annotated checklist of lichens, lichenicolous and allied fungi of Channel Islands National Park. Opuscula Philolichenum. 11: $145-302$.

Kondratyuk S, Khodosovtsev A, Zelenko S. 1998. The Second Checklist of Lichen Forming, Lichenicolous and Allied Fungi of Ukraine. Kiev: Phytosociocentre $179 \mathrm{p}$.

Kondratyuk SY, Popova LP, Lackovicova A, Pišút I. 2003. A catalogue of the Eastern Carpathian Lichens. KievBratislava: M.H. Kholodny Institute of Botany.

Nash TH, Ryan BD, Gries C, Bungartz F. (eds.) 2004. Lichen Flora of the Greater Sonoran Desert Region. Vol 2. Lichens Unlimited, Arizona State University, Tempe, Arizona.

Nasim G, Iqbal SH, Mirza JH, Riaz T, Butt S, Anjum T, Shabbir A. 2004. A brief note of lichens from Swat District. Mycopathologia. 2: 79-82.

Oxner AM. 1968. Flora of the lichens of Ukraine. Kyiv: Naukova dumka $498 \mathrm{pp}$.

Sambrook J, Russell DW. 2001. Detection of DNA in agarose gels. Molecular Cloning, A Laboratory Manual, (3rd Ed.) Cold Spring Harbor Laboratory Press, New York 5-14.

Schmull M, Miadlikowska J, Pelzer M, Stocker-Wörgötter E, Hofstetter V, Fraker E, Kauff F. 2011. Phylogenetic affiliations of members of the heterogeneous lichen- 
forming fungi of the genus Lecidea sensu Zahlbruckner (Lecanoromycetes, Ascomycota). Mycologia. 103 (5): 983-1003.

Singh KP, Sinha GP. 2010. Indian lichens: an annotated checklist. Kolkata: Botanical Survey of India.

Smith CW, Aptroot A, Coppins BJ, Fletcher A, Gilbert OL, James PW, Wolseley PA. (eds.) 2009. The Lichens of Great Britain and Ireland. Natural History Museum Publications, in association with The British Lichen Society, London.

Tamura K, Stecher G, Peterson D, Filipski A, Kumar S. 2013. MEGA6: molecular evolutionary genetics analysis version 6.0. Molecular Biology and Evolution. 30 (12): 2725-2729.

Vondrák J, Palice Z, Khodosovtsev A, Postoyolkin S. 2010. Additions to the diversity of rare or overlooked lichens and lichenicolous fungi in Ukrainian Carpathians. Chornomors'k Bot Zhurnal. 6: 6-34.

White TJ, Bruns T, Lee SJWT, Taylor J. 1990 Amplification and direct sequencing of fungal ribosomal RNA genes for phylogenetics. PCR protocols: a guide to methods and applications 18 (1): 315-322.

Zhao X, Zhang LL, Zhao ZT, Wang WC, Leavitt SD, Lumbsch HT. 2015. A molecular phylogeny of the lichen genus Lecidella focusing on species from mainland China. PloS One. 10 (9): e0139405 